\title{
The Study of Mucosal-Associated Invariant T Cells in Colon Cancer and Roles in Immune Activities
}

\author{
Juanfen Mo' \\ Li Zheng' \\ Zhenzhen $\mathrm{Gao}^{2}$ \\ Jiayuan $\mathrm{Wu}^{\prime}$ \\ Yi Baol,2
}

'The Key Laboratory, The Second Affiliated Hospital of Jiaxing University, Jiaxing, Zhejiang, 3I4000, People's Republic of China; ${ }^{2}$ Department of Oncology, The Second Affiliated Hospital of Jiaxing University, Jiaxing, Zhejiang, 314000, People's Republic of China
Correspondence: Yi Bao

The Key Laboratory, The Department of Oncology, The Second Hospital of Jiaxing, I5I8 Huanchen North Road, Zhejiang, 314000, People's Republic of China

Tel +865 7382073185

Fax +8657382082936

Email ybao201 I@gmail.com
Introduction: Mucosal-associated invariant T (MAIT) cells are a group of unconventional $\mathrm{T}$ cells, which strongly express CD161 and are involved in defending against infectious pathogens and inflammatory diseases. They are activated by inflammatory cytokines, secrete various cytokines and cytotoxic molecules, and express chemokine receptors and integrins. However, the underlying mechanisms of MAIT cells in colon cancer are still not fully understood.

Methods: The phenotype and frequency of circulating MAIT cells were investigated by flow cytometry in colon cancer patients and healthy donors. CD161 was examined in cancerous and paracancerous nontumor tissues of colon cancer patients by immunohistochemistry. The serum levels of IFN- $\gamma$ and IL-17A were analyzed by ELISA. Finally, MAIT cells were also detected in peripheral blood and tumor tissues in a CT26 tumor-bearing mice model.

Results: The percentages of $\mathrm{CD}^{+} \mathrm{CD}^{-}$MAIT cells, $\mathrm{CD} 4^{-} \mathrm{CD} 8^{+}$MAIT cells, and $\mathrm{CD} 4^{-} \mathrm{CD} 8^{-}$MAIT cells increased in the peripheral blood of colon cancer patients compared with healthy donors. The expression of CD161 protein in colon cancer cancerous tissues was higher than that in the paracancerous nontumor tissues. The killer cell lectin-like receptor B1 (KLRB1), a coding gene for CD161, was positively associated with the gene expressions of immune inhibitory receptors, such as CTLA4, HAVCR2, PDCD1, and CD274 in colon cancer. Furthermore, the serum levels of IFN- $\gamma$ and CEA were positively correlated with $\mathrm{CD} 8^{+}$MAIT cells in the peripheral blood of colon cancer patients.

Conclusion: Taken together, our data suggest that the circulating MAIT cells and the expression of CD161 protein in the tumor tissues increased in colon cancer patients, and MAIT cells may participate immune activities in colon cancer.

Keywords: MAIT cells, colon cancer, KLRB1, inflammation, immune activities

\section{Introduction}

Colon cancer is one of the most common human malignant diseases and it is the third-leading cause of cancer-related deaths worldwide. ${ }^{1}$ Metastasis is the most frequent cause of death in colon cancer patients. T lymphocytes, natural killer (NK) cells, B lymphocytes, and macrophages from tumor microenvironments are important determinants for cancer occurrence and progression and ultimately affect clinical outcomes, by interacting crosstalking with tumor cells.

Mucosal-associated invariant T (MAIT) cells are a subset of "unconventional" $\mathrm{T}$ lymphocytes that are highly abundant in human liver tissues, mucosal tissues, and peripheral blood. $^{2-4}$ MAIT cells express semi-invariant T-cell receptor (TCR) $\alpha$ chains (V $\alpha 7.2-\mathrm{J} \alpha 33$ in humans and V $\alpha 19-\mathrm{J} \alpha 33$ in mice) and TCR $\beta$ chains (V $\beta 2$, $\mathrm{V} \beta 13$, and $\mathrm{V} \beta 22$ in humans and $\mathrm{V} \beta 6$ and $\mathrm{V} \beta 8$ in mice). ${ }^{5,6}$ In addition, MAIT cells 
are recognized by high expressions of CD161, CD8, CD4, and $\mathrm{CD}^{-} \mathrm{CD}^{-}$double negative (DN). ${ }^{7}$ They can be stimulated by microbial metabolites and exhibit rapid effector responses by the major histocompatibility complex (MHC) class I-like protein (MR1). Activated MAIT cells can produce tumor necrosis factor- $\alpha$ (TNF- $\alpha)$, interferongamma (IFN- $\gamma$ ), interleukin-17A (IL-17A), and upregulation of intracellular granzyme B (GrB), then exhibit rapid, innate-like effector responses. ${ }^{8}$ MAIT deficient mice have a higher microbial burden, lower tissue cytokine production, and higher mortality in sepsis. ${ }^{9}$

MAIT cells are implicated in the association of malignancies with both favorable and unfavorable outcomes in tumor immunology. Some scholars have pointed out that MAIT cells are accumulated in the tumor environment in colon cancer patients leading to cancer cell cycle arrest by secreting IFN- $\gamma$ and IL-17. ${ }^{10}$ On the other hand, higher MAIT cells infiltrating in colorectal tumors have been reported to be associated with a poor clinical outcome. ${ }^{11}$ Tumor-infiltrating MAIT cells have an exhausted phenotype and reduced IFN- $\gamma$ and $\mathrm{GrB}$ secretion, suggesting impaired anti-tumor immunities in colon cancer patients. ${ }^{12}$ Due to the controversial studies, it is still necessary to further explore the other roles of MAIT cells in colon cancer.

In this study, we firstly examined the phenotype and proportion of circulating MAIT cells in colon cancer patients, and the expression of CD161, a highly positive marker on the surface of MAIT, was also explored in colon cancer tumor tissues. The correlations of MAIT cells with other immunosuppressive cell markers and inflammatory factors were also investigated. Finally, we established a CT26 tumor-bearing mice model and further examined the MAIT cells in this animal model. Our results indicate that MAIT cells increase in colon cancer patients and are potential associated with the immune activities of colon cancer.

\section{Materials and Methods}

\section{Patients and Samples}

A total of 47 colon cancer patients (27 males and 20 females, aged from 45 to 81 years old, median age 63 years old) were recruited in this study who hospitalized in the Second Affiliated Hospital of Jiaxing University between 2018 Jul to 2020 Dec. Among them, serums were collected from all patients and used for ELISA detection. The fresh whole venous blood were obtained from 20 of the 47 patients and used for Flow cytometry. Paraffin-embedded tumor tissues and matched paracancerous tissues were collected from 12 colon cancer patients. All tumor samples used in this study were pathologically diagnosed as colon adenocarcinoma. Peripheral blood and serum samples were obtained from 20 healthy donors from the physical examination center in the Second Affiliated Hospital of Jiaxing University (12 males and 8 females, aged from 42 to 75 years old, median age 56 years old). This study was approved by the ethics committees of the Second Affiliated Hospital, Jiaxing University (Ethics number: jxey-2018038), which accorded with the ethical standards formulated in the Helsinki Declaration. All the patients offering clinical materials were signed the informed consents. Additional patient data were present in Table 1.

\section{Flow Cytometry}

The human fluorescent-labeled antibodies included PerCPCy5.5-anti-CD3, FITC-anti-CD4, APC-Cy7-anti-CD8, APC-anti-CD161 and PE-anti-TCR Va7.2. The mouse fluorescent-labeled antibodies included FITC-anti-CD3, APC-Cy7-anti-CD8, APC-anti-CD24 and PE-anti-TCR $\beta$. All antibodies were purchased from BD Biosciences (BD, USA). For extracellular staining of immune markers, $50 \mu \mathrm{L}$ of peripheral blood were stained with fluorescentlabeled antibodies, and incubated on ice for 20 minutes. Isotype-matched control antibodies were used as negative controls. After centrifugation, the cells were made into a suspension and then quantified by FACS Canto II (BD

Table I Demographic, Sex, and Clinical Stages of Patients with Colon Cancer and Healthy Donors

\begin{tabular}{|l|l|l|}
\hline & Healthy Donors & Colon Cancer Patients \\
\hline Total samples & 20 & 47 \\
\hline Age (years) & & \\
Min & 42 & 45 \\
Max & 75 & 81 \\
Mean & $55.60 \pm 2.24$ & $62.62 \pm 1.46$ \\
\hline Sex & & \\
Male & 12 & 27 \\
Female & 8 & 20 \\
\hline Clinical stages & & \\
Stage I-II & - & 16 \\
Stage III-IV & - & 31 \\
\hline
\end{tabular}


Biosciences, USA). A total of 50000 events were recorded and analyzed by the FACS Canto II diva software (BD Biosciences, USA). The data were analyzed by FlowJo v7.6.2 software (Tree Star, Ashland, OR).

\section{Immunohistochemistry}

Paraffin-embedded tumor tissues were sectioned at $5 \mu \mathrm{m}$ thickness, followed by being dewaxed and rehydrated. After washing in phosphate-buffered saline (PBS), nonspecific antigens were prevented by incubation of the slides with $1 \%$ normal goat serum for $20 \mathrm{~min}$. Then the slides were incubated with 1:200 diluted anti-CD161 antibody (ab197979, Abcam, USA) at $4{ }^{\circ} \mathrm{C}$ for overnight. Slides were treated with secondary antibody anti-rabbit IgG (Servicebio, China) for $30 \mathrm{~min}$ and then incubated with simple stain DAB solution (Servicebio, China). Finally, slides were counterstained with hematoxylin, dehydrated, and mounted.

The slides were observed and photographed by a light microscope using $1 \times 100$ and 200 magnification (Zeiss, Germany).

\section{Enzyme Linked Immunosorbent Assay}

The concentrations of IFN- $\gamma$ and IL-17A in the human serums were analyzed by using an ELISA methods with commercially available kits (Sizhengbai Biotech, Beijing, China). ELISA experiments were performed according to manufacturer's protocols.

\section{TCGA and GEPIA Database Analysis}

The gene expressions of KLRB1 in tumor tissues, normal tissues, as well as different TNM stages of tumor tissues were studied by analyzing colon cancers of the TCGA database (https://cancergenome.nih.gov/). The correlation of KLRB1 with cytotoxic $\mathrm{T}$ lymphocyte-associated antigen-4 (CTLA4), hepatitis A virus cellular receptor 2 (HAVCR2; gene coded TIM-3 protein), programmed cell death 1 (PDCD1; gene coded PD-1 protein), and CD274 were determined by the data set of GEPIA (http://gepia. cancer-pku.cn/).

\section{Animal Experiments}

8-week-old female BALB/c mice were purchased from Shanghai Laboratory Animal Center, Chinese Academy of Sciences (SLAC, CAS).

$\mathrm{BALB} / \mathrm{c}$ mice were inoculated subcutaneously in a single-cell suspension with $2.5 \times 10^{5}$ CT26 tumor cells. Tumor volumes were measured every two days until 3 weeks by caliper using the following formula $\left(\right.$ length $\times$ width $\left.^{2}\right) / 2$. After 3 weeks, the mice were sacrificed. Tumor tissues were washed with PBS and isolated into mononuclear cells by a gentleMACS Dissociator (Miltenyi, Germany). The whole blood and single-cell fractions of tumor tissues were analyzed by flow cytometry within $4 \mathrm{~h}$ collection. All animal experiments were followed by the guidelines of the Administration of Affairs Concerning Experimental Animals (State Scientific and Technological Commission, P. R. China, 1988) for the welfare and treatment of the laboratory animals. The experiments were approved by the Animal Ethics Committee of Jiaxing University, Jiaxing, China.

\section{Statistical Analysis}

All experimental data were expressed as the mean \pm standard deviation (S.D.). Statistical analyses were performed using GraphPad Prism 6 (GraphPad Software, USA). Statistical significance of expression changes were calculated by unpaired $t$-test for two groups. The two-way ANOVA was employed to compare grouped and paired data sets as appropriate. Correlations were analyzed using the Spearman's rank correlation test. Differences with a $P$ $<0.05$ were considered a statistically significant.

\section{Results}

\section{MAIT Cells phenotype and Frequency in the Peripheral Blood of Colon Cancer Patients}

The phenotype and frequency of MAIT cells were evaluated in fresh peripheral blood of colon cancer patients and healthy donors by flow cytometry. MAIT cells were verified by using CD3, CD4, CD8, CD161, and TCR V 27.2 antibodies, as previously established by Rouxel et al. ${ }^{13}$ The gating strategy was shown to identify $\mathrm{CD} 4^{-} \mathrm{CD} 8^{+}$ MAIT cells, $\mathrm{CD}^{+}{ }^{+} \mathrm{CD} 8^{-}$MAIT cells, and $\mathrm{CD} 4^{-} \mathrm{CD} 8^{-}$ MAIT cells in human blood as Figure 1A. We observed that the percentage of $\mathrm{CD} 4^{-} \mathrm{CD} 8^{+}$MAIT cells frequency in the peripheral blood of colon cancer patients (11.63 \pm $1.52 \%, \mathrm{~N}=20$ ) was significantly higher compared with healthy donors $(5.94 \pm 1.13 \%, \mathrm{~N}=20)(P<0.05)$ (Figure 1B). Moreover, $\mathrm{CD}^{-} \mathrm{CD} 8^{-}$MAIT cells frequency in the peripheral blood of colon cancer patients $(4.56 \pm$ $1.12 \%)$ was significantly higher compared with healthy donors $(3.61 \pm 1.16 \%)(P<0.05)$ (Figure $1 \mathrm{C})$. $\mathrm{CD} 4^{+} \mathrm{CD} 8^{-}$MAIT cells in the peripheral blood of colon cancer patients $(0.56 \pm 0.09 \%)$ was significantly higher 
A

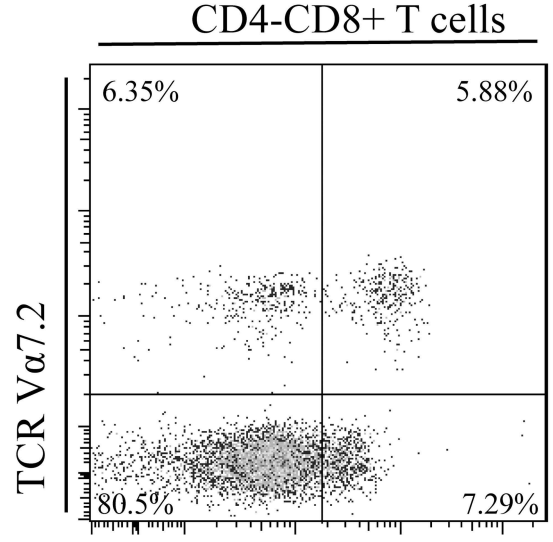

CD161

B

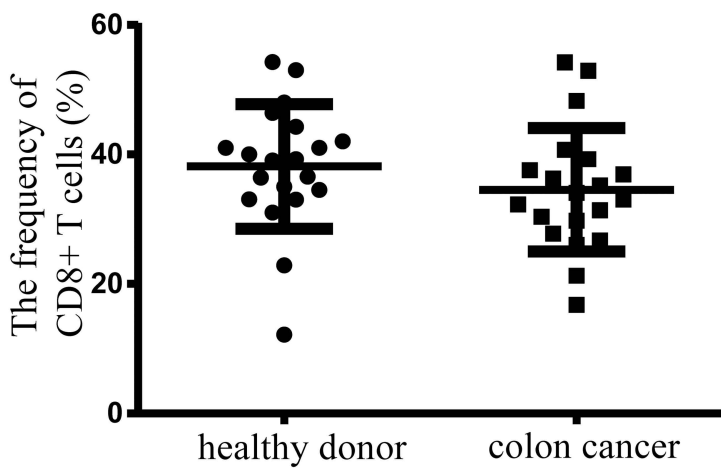

C

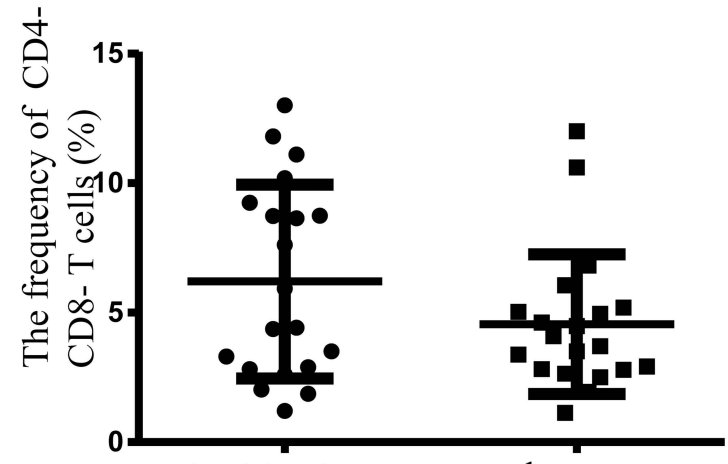

healthy donor

D

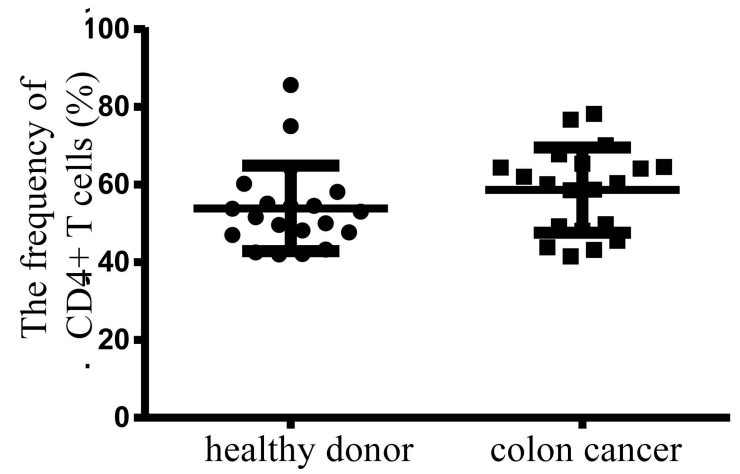

CD4-CD8- T cells

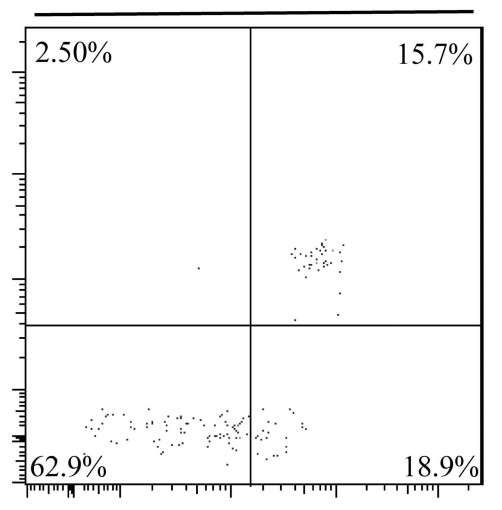

CD4+CD8- T cells

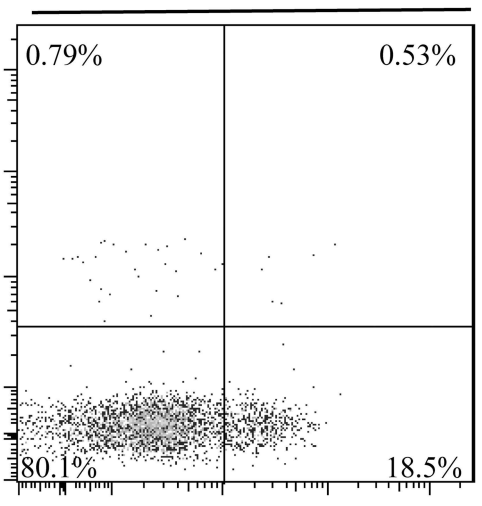

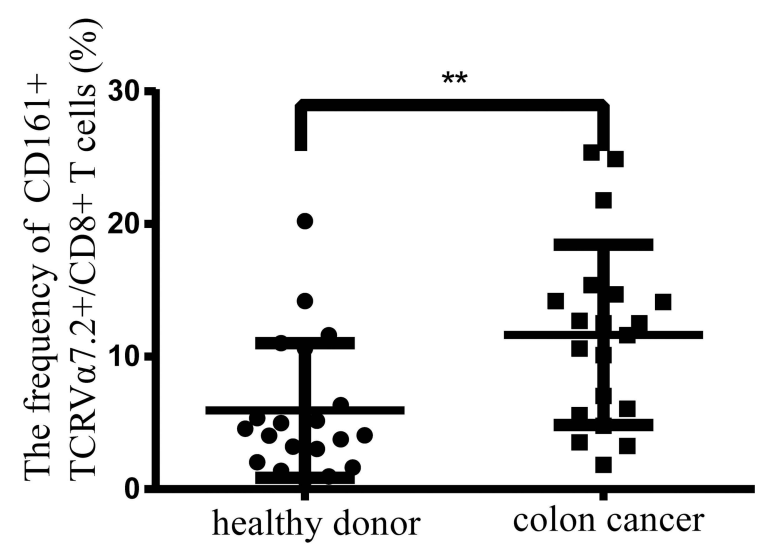
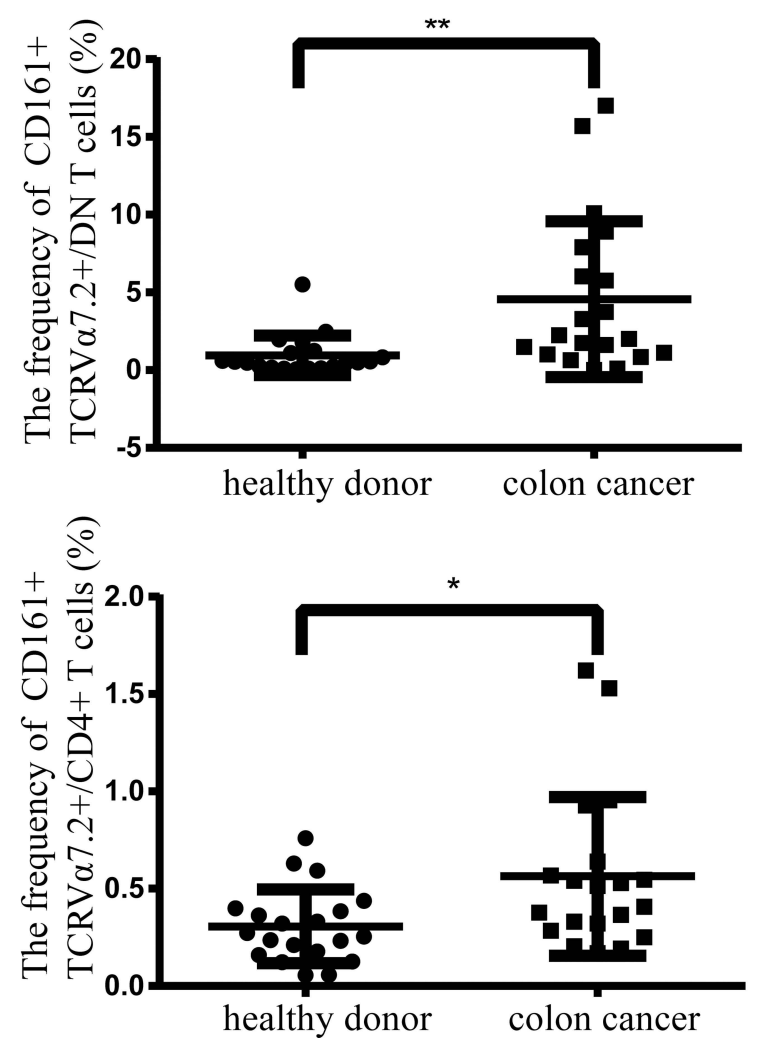

Figure I Frequency and phenotype of MAIT cells in the peripheral blood of colon cancer patients and healthy donors. (A) CDI6I ${ }^{+} \mathrm{TCR}$ V $\alpha 7.2^{+}$cells were gated on

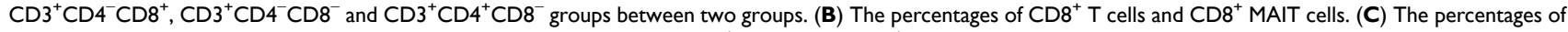
$\mathrm{CD} 4^{-} \mathrm{CD} 8^{-} \mathrm{T}$ cells and $\mathrm{CD} 4^{-} \mathrm{CD} 8^{-}$MAIT cells. (D) The percentages of CD4 $4^{+} \mathrm{T}$ cells and CD4 ${ }^{+}$MAIT cells. Data were presented as mean $\pm \mathrm{SD}$. $* P<0.05$, $* * P<0.0 \mathrm{I}$, significantly different from the values in the healthy donors. 

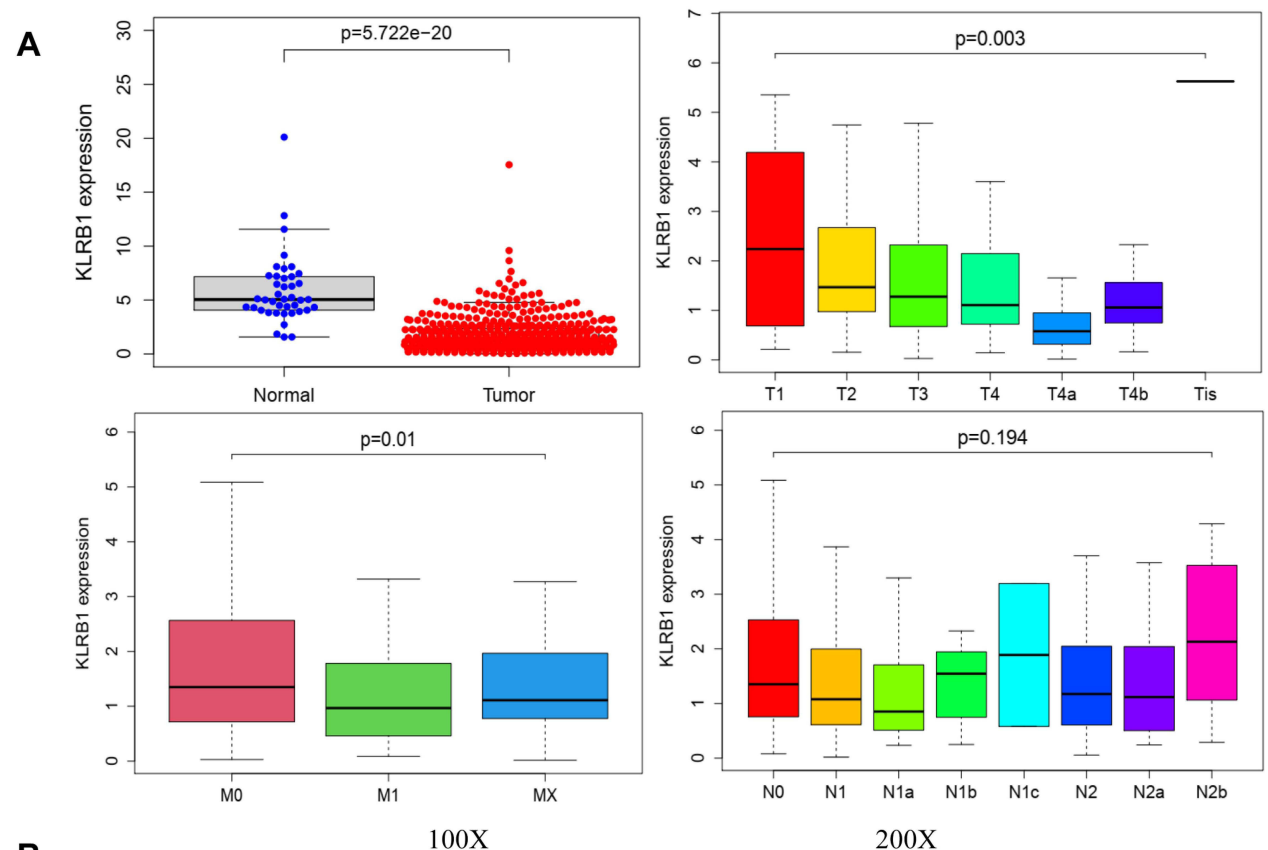

B
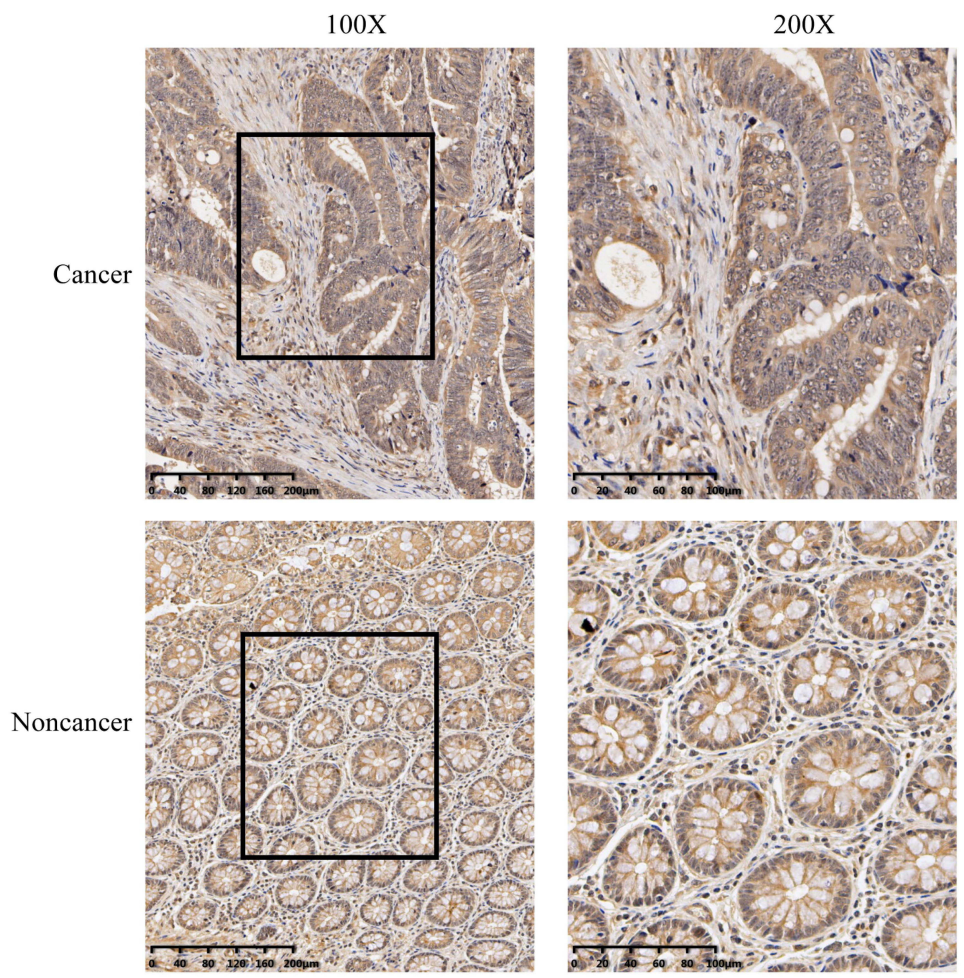

C

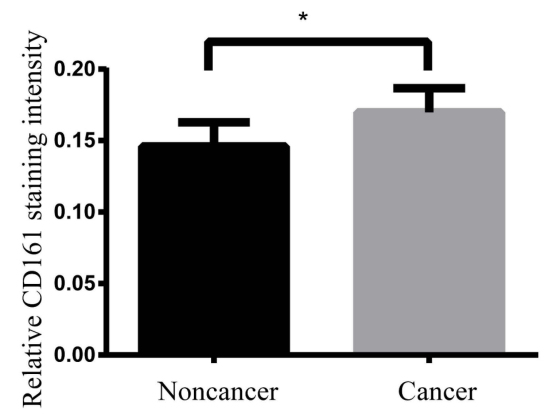

Figure 2 Continue. 

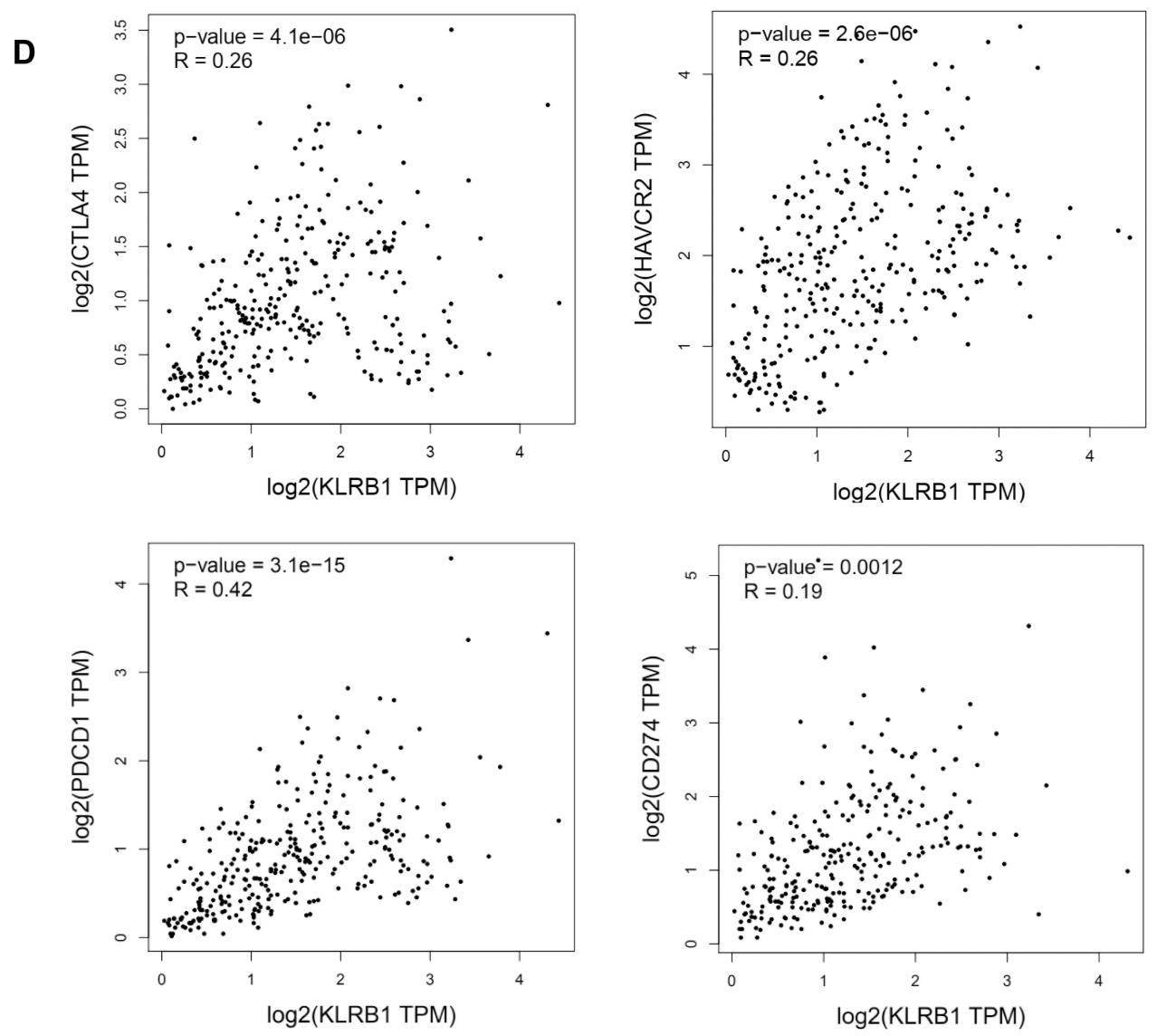

Figure 2 Expression of CDI6I in noncancerous and cancer tissues of colon cancer. (A) The mRNA expression of KLRBI in colon cancer tumor tissues and normal tissues, and different clinical TNM stages of colon cancer tumor tissues by the TCGA database. (B) Comparison of CDI6I in noncancerous and cancer tissues via IHC staining. Scale bars: $\times 100, \times 200 .(C)$ Quantitative analysis of CDI6I staining intensity in cancer tissues and noncancerous tissues by Image J. (D) Correlation of KLRBI with CTLA4, HAVCR2, PDCDI, CD274 by the GEPIA. $* P<0.05$, significantly different from the values in the healthy donors.

compared with healthy donors $(0.31 \pm 0.04 \%)(P<0.05)$ (Figure 1D). However, the percentages of circulating $\mathrm{CD} 4^{-} \mathrm{CD} 8^{+} \mathrm{T}$ cells, $\mathrm{CD} 4^{-} \mathrm{CD} 8^{-} \mathrm{T}$ cells, and $\mathrm{CD} 4^{+} \mathrm{CD} 8^{-}$ $\mathrm{T}$ cells were no significant difference between colon cancer patients and healthy donors $(P>0.05)$ (Figure 1B-D).

\section{KLRBI mRNA and CDI6I Protein Expression in Colon Cancer Tissues}

CD161 is coded by gene KLRB1. The mRNA levels of KLRB1 in colon cancer tumor tissues and normal tissues were compared by using TCGA databases. The mRNA levels of KLRB1 in colon cancer tumor tissues were decreased compared with normal tissues $(P<0.05)$ (Figure 2A). In different TNM stages of colon cancer tissues, KLRB1 gene expression was highest at the T1 stage and the M1 stage $(P<0.05)$, lowest at the T4 stage $(P<0.05)$, whereas there was no significant difference at the $\mathrm{N}$ stages $(P>0.05)$ (Figure $2 \mathrm{~A})$. Then, we examined the CD161 protein expression in colon cancer tumor tissues and patient-matched normal mucosa tissues (noncancer) by IHC. The results showed that CD161 protein was markedly expressed in immune cells of colon cancer tumor tissues, while weakly expressed in noncancerous tissues (Figure 2B). After quantified by Image $\mathrm{J}$ software, the staining intensity of CD161 was statistically higher in colon cancer tumor tissues than that of noncancerous tissues $(P<0.05)$ (Figure $2 \mathrm{C}$ ). Moreover, KLRB1 was positively correlated with the genes of immune inhibitory receptors, such as CTLA4, HAVCR2, PDCD1, and CD274 by using GEPIA $(P<0.05)$ (Figure 2D).

\section{Correlation Between Circulating MAIT Cells and Serum Cytokines in Colon}

\section{Cancer Patients}

IFN- $\gamma$ and IL-17A were higher in the serum of colon cancer patients than those in healthy donors respectively (IFN- $\gamma: 9.64 \pm 1.30 \mathrm{pg} / \mathrm{mL}$ VS $2.18 \pm 0.50 \mathrm{pg} / \mathrm{mL}$; IL- 
A
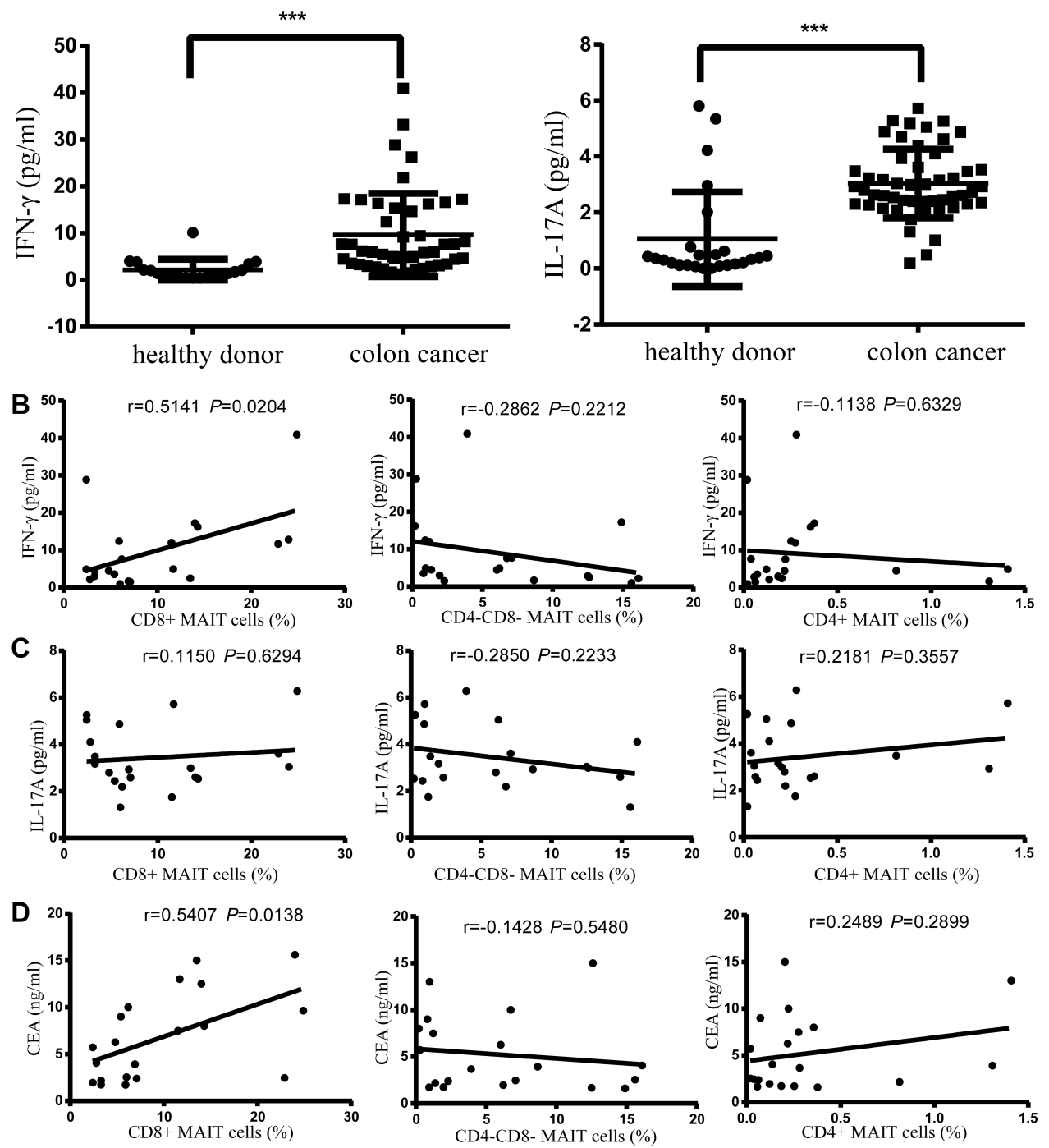

Figure 3 Serum cytokine levels in colon cancer patients and their correlation with circulating MAIT cells in colon cancer patients. (A) The serum levels of IFN- $\gamma$ and IL-I7A in colon cancer patients compared with healthy donors. (B) Correlation between serum IFN- $\gamma$ level and three subtypes of circulating MAIT cells in colon cancer patients. (C) Correlation between serum IL-I7A and three subtypes of circulating MAIT cells frequency in colon cancer patients. (D) Correlation between serum CEA concentration and three subtypes of circulating MAIT cells frequency in colon cancer patients. Differences between groups were analyzed using t-test. Correlation analysis was performed using the Spearman's rank correlation test. $* * * P<0.001$, significantly different from the values in the healthy donors.

17A: $3.04 \pm 0.17 \mathrm{pg} / \mathrm{mL}$ VS $1.04 \pm 0.34 \mathrm{pg} / \mathrm{mL})(P<$ 0.05 ) (Figure $3 \mathrm{~A}$ ). In addition, the serum levels of IFN- $\gamma$ were positively associated with circulating $\mathrm{CD}^{+}$MAIT cells in colon cancer patients $(P<0.05)$, whereas there was no significant difference in the $\mathrm{CD} 4^{-} \mathrm{CD} 8^{-}$MAIT cells and $\mathrm{CD}^{-}$MAIT cells $(P>0.05)$ (Figure $\left.3 \mathrm{~B}\right)$. The serum IL-17A level was not significantly associated with the three subtypes of circulating MAIT cells frequency in colon cancer patients $(P>0.05)$ (Figure 3C). The serum levels of CEA were positively associated with circulating $\mathrm{CD}^{+}$MAIT cells in colon cancer patients $(P<0.05)$, whereas there was no significant difference in the
$\mathrm{CD}^{-} \mathrm{CD}^{-}$MAIT cells and $\mathrm{CD}^{-}$MAIT cells $(P>$ 0.05) (Figure 3D).

\section{The MAIT Cells in the Peripheral Blood and Single-Cell Fractions of Tumor Tissues in a CT26 Tumor-Bearing Mice Model}

To observe the MAIT cells in mice, we constructed a CT26 tumor-bearing mice model. BALB/c mice were inoculated subcutaneously with $2.5 \times 10^{5} \mathrm{CT} 26$ cells. We regarded the first day of injection as day 0 , then continued feeding for 
A

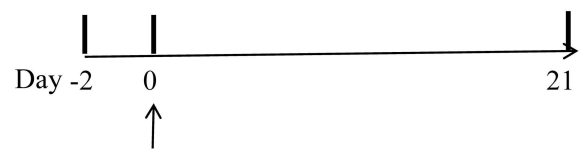

Subcutaneously injection of $2.5 \times 10^{5}$ CT26 colorectal tumor cells
B

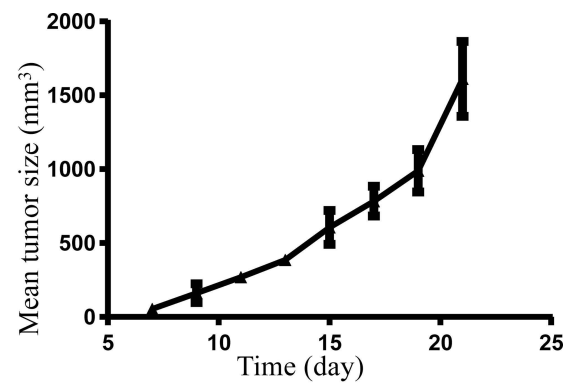

C

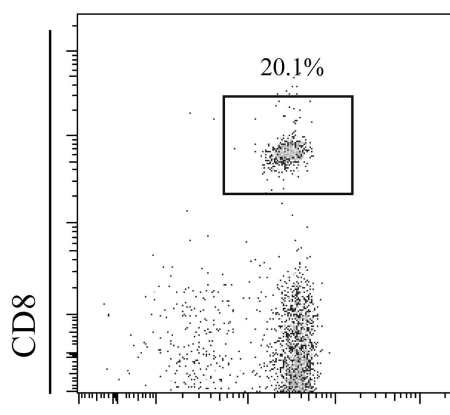

CD3

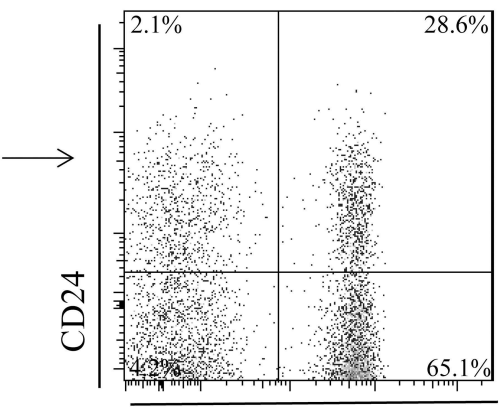

TCR $\beta$

D
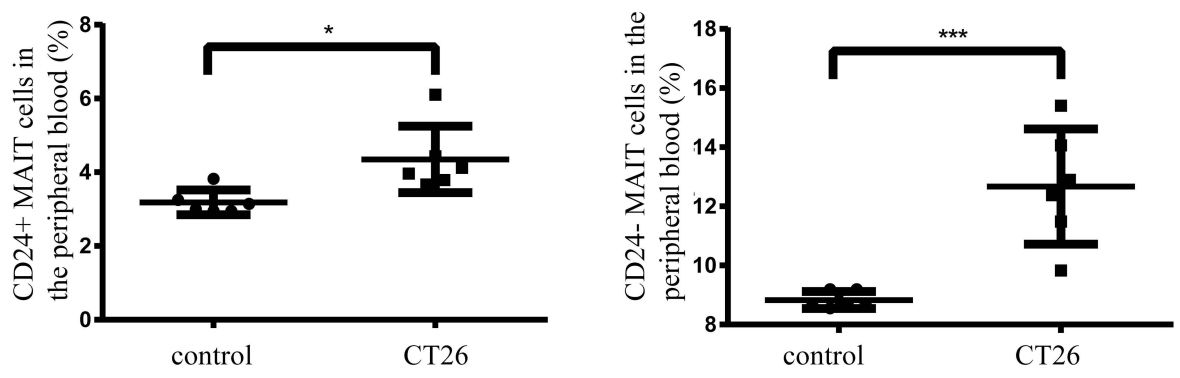

E

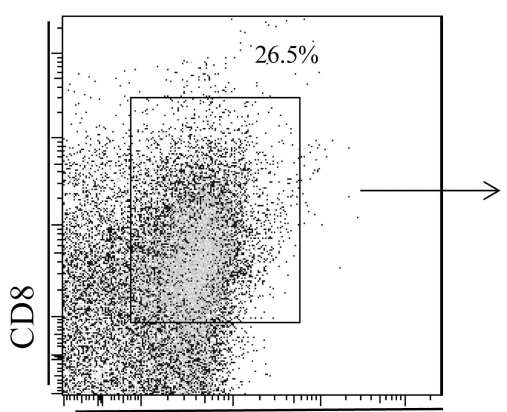

CD3

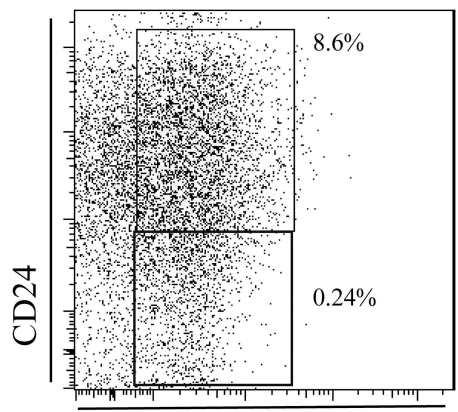

TCR $\beta$

Figure 4 Frequency and phenotype of MAIT cell in the CT26 tumor-bearing mice. (A) Schematic diagram of an experimental protocol. $2.5 \times 10^{5}$ CT26 cells were injected into BALB/c mice on day 0 , then continued feeding for 3 weeks. (B) Growth curves of tumor of CT26 tumor-bearing mice. (C) CD24 ${ }^{+}$TCR $\beta^{+}$cells and CD24 TCR $\beta^{+}$cells were gated on the $\mathrm{CD} 3^{+} \mathrm{CD} 8^{+}$group in the peripheral blood of $\mathrm{CT} 26$ tumor-bearing mice and controls. (D) Increased frequencies of $\mathrm{CD} 3^{+} \mathrm{CD} 8^{+} \mathrm{CD} 24^{+} \mathrm{TCR} \beta^{+} \mathrm{MAIT}$ cells and $\mathrm{CD} 3^{+} \mathrm{CD} 8^{+} \mathrm{CD} 24^{-} \mathrm{TCR} \beta^{+}$MAIT cells in the peripheral blood of CT26 tumor-bearing mice and controls. (E) CD24 ${ }^{+} \mathrm{TCR} \beta^{+}$cells and CD24 $4^{-} \mathrm{TCR} \beta^{+}$cells were gated on $\mathrm{CD}^{+} \mathrm{CD}^{+}$group in single-cell fractions of mice tumor tissues. Data were presented as mean $\pm \mathrm{SD}, \mathrm{N}=6$. $* P<0.05$, $* * * P<0.00 \mathrm{I}$, significantly different from the values in the controls.

mice until 21 days (Figure 4A). The tumor volumes were increasing linearly from day 0 to day 21 (Figure 4B). The antibodies of $\mathrm{CD} 3, \mathrm{CD} 8, \mathrm{CD} 24$, and TCR $\beta$ were used to identify MAIT cells in mice, as previously established by
Rahimpour et $\mathrm{al}^{14}$ (Figure 4C). The frequency of $\mathrm{CD}^{+} \mathrm{CD} 8^{+} \mathrm{CD} 24^{+} \mathrm{TCR} \beta^{+}$cells was significantly higher in the fresh peripheral blood of CT26 tumor-bearing mice $(4.35 \pm 0.37 \%, \mathrm{~N}=6)$ compared with control mice $(3.19 \pm$ 
$0.14 \%, \quad \mathrm{~N}=6$ ), and the frequency of $\mathrm{CD}^{+}{ }^{+} \mathrm{CD} 8{ }^{+} \mathrm{CD} 24^{-} \mathrm{TCR} \beta^{+}$cells was also higher in $\mathrm{CT} 26$ tumor-bearing mice $(12.67 \pm 0.80 \%, \mathrm{~N}=6)$ compared with control mice $(8.83 \pm 0.12 \%, \mathrm{~N}=6)(P<0.05)$ (Figure 4D). Subsequently, we isolated the single-cell fractions of tumor tissues from the CT26 tumor-bearing mice, CD24 $4^{+}$MAIT cells and CD24- MAIT cells were detected in the tumor tissues by flow cytometry (Figure 4E).

\section{Discussion}

In this study, we detected a higher frequency of MAIT cells in the peripheral blood of patients with colon cancer than that of healthy donors. The CD161 protein was highly expressed in colon cancer cancerous tissues compared with paracancerous nontumor tissues. Additionally, the $\mathrm{CD}^{+} \mathrm{CD} 8^{+} \mathrm{CD} 24^{+} \mathrm{TCR} \beta^{+}$ cells and $\mathrm{CD}^{+}{ }^{+} \mathrm{CD} 8^{+} \mathrm{CD} 24^{-} \mathrm{TCR} \beta^{+}$cells were found in the peripheral blood and tumor tissues of a CT26 tumor-bearing mice model. In mice, the mapping of MAIT cell development has a three-stage sequence, the earliest cells (at stage 1) are immature MAIT cells which are defined as CD24 ${ }^{+}$MAIT cells, the mature MAIT cells of stage 2 and stage 3 are defined as CD24 ${ }^{-}$MAIT cells. ${ }^{8}$ Furthermore, the percentage of circulating MAIT cells was positively correlated with serum levels of IFN- $\gamma$ and CEA in the patients with colon cancer. These findings suggested that MAIT cells may have an important impact on colon cancer tumorigenesis and progression.

Based on the expression of co-receptors CD4 and CD8, MAIT cells are categorized as $\mathrm{CD} 4^{+} \mathrm{CD} 8^{-}$MAIT cells, $\mathrm{CD}^{-} \mathrm{CD}^{+}$MAIT cells, and $\mathrm{CD}^{-} \mathrm{CD}^{-}$MAIT cells. Different subsets of MAIT cells produce similar levels of cytokines, although $\mathrm{CD}^{+}$MAIT cells may produce slightly more cytokines than do $\mathrm{CD}^{-} \mathrm{CD}^{-}$MAIT cells. ${ }^{8}$ Our results showed that all of these three subsets of MAIT cells were significantly increased in the circulation of colon cancer patients. Meanwhile, the percentage of MAIT cells was also increased in the peripheral blood of CT26 tumor-bearing mice compared with controls, and MAIT cells were infiltrated into the tumor tissues. We considered that the increased circulating MAIT cells may be responses due to the inflammatory stimulation, and alternatively, the increased MAIT cells migrate into the tumor microenvironment (TME) and promote tumor progression. This finding is supported by a recent study which showed that the frequency of circulating $\mathrm{CD}^{+}$MAIT cells is significantly higher in colorectal cancer patients. ${ }^{15}$ Another report showed that circulating MAIT cells were significantly increased in cervical cancer patients. ${ }^{16}$
Conversely, conflicted data were reported by another study, suggesting that MAIT cells highly accumulated in tumor tissues of colon cancer, but decreased in circulation, caused by redistribution of peripheral MAIT cells into TME. ${ }^{12}$ Whether MAIT cells act positive or negative roles in the peripheral blood of colon cancer remains debates and needs further work to elucidate the precise mechanisms.

In humans, CD161 is encoded by the KLRB1 gene and highly expresses on the surface of MAIT cells and NK cells. ${ }^{17}$ Through analyzing the TCGA database, the gene expression of KLRB1 is lower in the tumor tissues of colon cancer patients, but its expression appears to be higher in the earlier stage of colon cancer. Interestingly, CD161 protein was highly expressed in the peripheral blood and in tumor tissues of colon cancer patients, which is discordant with its mRNA study. The inconsistent expressions of a coding gene and a protein are present in some circumstance, the degradation pathways of a certain protein were dysregulated, thus, the underlying mechanisms are still needed to be further clarified. CD161 is considered to be an inhibitory receptor for tumor-specific $\mathrm{T}$ cells in glioma, and inhibition of CD161 receptor can enhance T cell-mediated killing of glioma cells and their anti-tumor function. ${ }^{18}$ Targeting the CD161 receptor may offer a new approach for enhancing the efficacy of immunotherapy in colon cancer. Moreover, KLRB1 is significantly associated with the genes of immune inhibitory receptors, such as CTLA4, HAVCR2, PDCD1, and CD274. These molecules are recognized as immune checkpoints and are associated with immunosuppressive signals in the T cells of TME in a variety of cancers, including lung cancer, colon cancer, breast cancer, and melanoma. $^{19,20}$ HAVCR2, also known as TIM-3, is expressed on immune cells with enhanced suppressive functions, and is related to a poor disease prognosis in various cancers. ${ }^{21}$ Interestingly, abundant MAIT cells are likely to make the anti-PD-1 treatment more effective in human metastatic melanoma. ${ }^{22}$ Therefore, MAIT cells may be considered a biomarker for treating patients with immune checkpoint inhibitors. In this study, we speculated that MAIT cells might influence the colon cancer progression through overexpression of CD161 on the surface of MAIT cells, which was associated with the aggravation of tumor immunosuppression, nevertheless, the precise mechanisms are still needed to be further investigated. For instance, whether blocking the signaling molecules to costimulate MAIT cells during CD161 engagement 
relieves the immunosuppressive activities of MAIT cells in colon cancer patients, is required in the following studies.

Previous studies have demonstrated that activated MAIT cells can secrete several cytokines, including IFN- $\gamma$ and IL17. ${ }^{8,23,24}$ Our results showed that the serum levels of IFN- $\gamma$ and IL-17A were elevated in colon cancer patients, suggesting that they may be associated with the inflammation-related tumor. The circulating $\mathrm{CD} 8^{+}$MAIT cells were significantly correlated with serum IFN- $\gamma$ and CEA, but not with $\mathrm{CD} 4^{-} \mathrm{CD} 8^{-}$MAIT cells and $\mathrm{CD}^{+}$MAIT cells. We propose that different subtypes have distinct functions, and the mechanism of this effect requires further study. In colon adenocarcinomas patients, MAIT cells highly accumulate in tumor tissues, particularly in hepatic metastatic tissues, with impaired IFN- $\gamma$ secretion by MAIT cells. ${ }^{25}$ It has been demonstrated that the ability of IFN- $\gamma$ secretion is impaired in tumor-infiltrating MAIT cells of metastatic colorectal cancer. ${ }^{15}$ However, it is unclear whether the MAIT cells in peripheral blood act the same way as in the TME. In addition, it remains to be determined whether the increased expression of CD161 at the T stage and M stage is associated with earlier stages colon cancer.

In summary, our study shows that the percentages of circulating MAIT cells are increased in colon cancer patients. The CD161 protein is highly expressed into tumor tissues of colon cancer patients, and the coding gene KLRB1 is significantly associated with immunosuppressive genes. Considering its positive correlation with serum levels of IFN- $\gamma$ and CEA, we hypothesize that MAIT cells may be involved in the inflammatory responses and immune activities in colon cancer. Although the direct and critical evidence needs to be proven, we still consider that the role of MAIT cells may be used as to serve as a marker for monitoring the antitumor immunity in colon cancer patients.

\section{Acknowledgments}

This work was supported by Science and Technology Bureau of Jiaxing, Zhejiang, China (2018AD32008 and 2020AY30015).

\section{Disclosure}

The authors report no conflicts of interest.

\section{References}

1. Bray F, Ferlay J, Soerjomataram I, et al. Global cancer statistics 2018: GLOBOCAN estimates of incidence and mortality worldwide for 36 cancers in 185 countries. CA Cancer J Clin. 2018;68(6):394 424. doi:10.3322/ caac. 21492
2. Gold MC, Cerri S, Smyk-Pearson S, et al. Human mucosal associated invariant T cells detect bacterially infected cells. PLoS Biol. 2010;8(6): e1000407. doi:10.1371/journal.pbio.1000407

3. Le Bourhis L, Martin E, Peguillet I, et al. Antimicrobial activity of mucosal-associated invariant $\mathrm{T}$ cells. Nat Immunol. 2010;11 (8):701-708. doi:10.1038/ni.1890

4. Dusseaux M, Martin E, Serriari N, et al. Human MAIT cells are xenobiotic-resistant, tissue-targeted, CD161 hi IL-17-secreting T cells. Blood. 2011;117(4):1250-1259. doi:10.1182/blood-2010-08-303339

5. Tilloy F, Treiner E, Park SH, et al. An invariant T cell receptor alpha chain defines a novel TAP-independent major histocompatibility complex class Ib-restricted alpha/beta $\mathrm{T}$ cell subpopulation in mammals. J Exp Med. 1999;189(12):1907-1921. doi:10.1084/jem.189.12.1907

6. Treiner E, Duban L, Bahram S, et al. Selection of evolutionarily conserved mucosal-associated invariant $\mathrm{T}$ cells by MR1. Nature. 2003;422(6928):164-169. doi:10.1038/nature01433

7. Reantragoon R, Corbett AJ, Sakala IG, et al. Antigen-loaded MR1 tetramers define $\mathrm{T}$ cell receptor heterogeneity in mucosal-associated invariant T cells. J Exp Med. 2013;210(11):2305-2320. doi:10.1084/ jem. 20130958

8. Godfrey DI, Koay HF, McCluskey J, et al. The biology and functional importance of MAIT cells. Nat Immunol. 2019;20(9):1110-1128. doi:10.1038/s41590-019-0444-8

9. Trivedi S, Labuz D, Anderson CP, et al. Mucosal-associated invariant $\mathrm{T}$ (MAIT) cells mediate protective host responses in sepsis. Elife. 2020;9. doi:10.7554/eLife.55615

10. Ling L, Lin Y, Zheng W, et al. Circulating and tumor-infiltrating mucosal associated invariant T (MAIT) cells in colorectal cancer patients. Sci Rep. 2016;6:20358. doi:10.1038/srep20358

11. Zabijak L, Attencourt C, Guignant C, et al. Increased tumor infiltration by mucosal-associated invariant $\mathrm{T}$ cells correlates with poor survival in colorectal cancer patients. Cancer Immunol Immunother. 2015;64(12):1601-1608. doi:10.1007/s00262-015-1764-7

12. Rodin W, Sundstrom P, Ahlmanner F, et al. Exhaustion in tumor-infiltrating Mucosal-Associated Invariant $\mathrm{T}$ (MAIT) cells from colon cancer patients. Cancer Immunol Immunother. 2021. doi:10.1007/s00262-021-02939-y.

13. Rouxel O, Da SJ, Beaudoin L, et al. Cytotoxic and regulatory roles of mucosal-associated invariant $\mathrm{T}$ cells in type 1 diabetes. Nat Immunol. 2017;18(12):1321-1331.

14. Rahimpour A, Koay HF, Enders A, et al. Identification of phenotypically and functionally heterogeneous mouse mucosal-associated invariant T cells using MR1 tetramers. J Exp Med. 2015;212 (7):1095-1108. doi:10.1084/jem.20142110

15. Sundstrom P, Ahlmanner F, Akeus P, et al. Human mucosa-associated invariant $\mathrm{T}$ cells accumulate in colon adenocarcinomas but produce reduced amounts of IFN-gamma. $J$ Immunol. 2015;195 (7):3472-3481. doi:10.4049/jimmunol.1500258

16. Lu Z, Zhu M, Marley JL, et al. The combined action of monocytic myeloid-derived suppressor cells and mucosal-associated invariant $\mathrm{T}$ cells promotes the progression of cervical cancer. Int $J$ Cancer. 2021;148(6):1499-1507. doi:10.1002/ijc.33411

17. Fergusson JR, Fleming VM, Klenerman P. CD161-expressing human T cells. Front Immunol. 2011;2:36. doi:10.3389/fimmu.2011.00036

18. Mathewson ND, Ashenberg O, Tirosh I, et al. Inhibitory CD161 receptor identified in glioma-infiltrating $\mathrm{T}$ cells by single-cell analysis. Cell. 2021;184(5):1281-1298. doi:10.1016/j. cell.2021.01.022

19. Ebert P, Cheung J, Yang Y, et al. MAP kinase inhibition promotes $\mathrm{T}$ cell and anti-tumor activity in combination with PD-L1 checkpoint blockade. Immunity. 2016;44(3):609-621. doi:10.1016/j. immuni.2016.01.024

20. Borst J, Busselaar J, Bosma D, et al. Mechanism of action of PD-1 receptor/ligand targeted cancer immunotherapy. Eur J Immunol. 2021;51(8):1911-1920. doi:10.1002/eji.202048994 
21. Andrews LP, Yano H, Vignali D. Inhibitory receptors and ligands beyond PD-1, PD-L1 and CTLA-4: breakthroughs or backups. Nat Immunol. 2019;20(11):1425-1434. doi:10.1038/s41590-019-0512-0

22. De Biasi S, Gibellini L, Lo TD, et al. Circulating mucosal-associated invariant $\mathrm{T}$ cells identify patients responding to anti-PD-1 therapy. Nat Commun. 2021;12(1):1669. doi:10.1038/s41467-021-21928-4

23. Ju JK, Cho YN, Park KJ, et al. Activation, deficiency, and reduced IFN-gamma production of mucosal-associated invariant $\mathrm{T}$ cells in patients with inflammatory bowel disease. J Innate Immun. 2020;12 (5):422-434. doi:10.1159/000507931
24. Shao C, Zhu C, Zhu Y, et al. Decrease of peripheral blood mucosal-associated invariant $\mathrm{T}$ cells and impaired serum Granzyme-B production in patients with gastric cancer. Cell Biosci. 2021;11(1):12. doi:10.1186/s13578-020-00518-9

25. Shaler CR, Tun-Abraham ME, Skaro AI, et al. Mucosa-associated invariant $\mathrm{T}$ cells infiltrate hepatic metastases in patients with colorectal carcinoma but are rendered dysfunctional within and adjacent to tumor microenvironment. Cancer Immunol Immunother. 2017;66 (12):1563-1575. doi:10.1007/s00262-017-2050-7

\section{Publish your work in this journal}

OncoTargets and Therapy is an international, peer-reviewed, open access journal focusing on the pathological basis of all cancers, potential targets for therapy and treatment protocols employed to improve the management of cancer patients. The journal also focuses on the impact of management programs and new therapeutic

Submit your manuscript here: https://www.dovepress.com/oncotargets-and-therapy-journal agents and protocols on patient perspectives such as quality of life, adherence and satisfaction. The manuscript management system is completely online and includes a very quick and fair peer-review system, which is all easy to use. Visit http://www.dovepress.com/ testimonials.php to read real quotes from published authors. 